\title{
Further preliminary findings on some effects of very fast sequential input rates on perception
}

\author{
M. S. MAYZNER, M. E. TRESSELT, A. J. ADRIGNOLO AND A. COHEN
}

NEW YORK UNIVERSITY

A previous study, employing a computer-based CRT display system, showed that if the visual system is presented with a string of very fast sequential inputs approximately the first half of these inputs will not be perceived if display order is irregular and display input rate is in the range from approximately 10 to $20 \mathrm{msec}$. per letter and per interval between letters. In the present study, this phenomena called sequential blanking is examined further and the results suggest certain irregular display orders may also give rise to a spatial displacement of one letter away from another in the sequence, and this phenomena has been called sequential displacement.

In a recent paper (Mayzner, Tresselt, \& Cohen, 1966), a study was made of the effects of presenting to the visual system a string of very fast sequential inputs, employing a computer-based CRT display system. In this study the basic procedure involved presenting a string of letters, one at a time, in adjacent spatial positions on a CRT display, in which display rate was varied from approximately $.25 \mathrm{msec}$. per letter and per interval between letters up to approximately $50 \mathrm{msec}$. Display order also was varied so as to yield sequences in which the letters were displayed in either regular orders (i.e., the display sequence of letter positions was always progressive from left to right or right to left) or irregular orders (i.e., the display sequence jumped irregularly from one letter position to another). The results showed that for either five- or 10-letter sequences approximately the first half of these sequentially presented letters were not perceived, if display order was irregular and display input rate fell in the range from approximately 10 to $20 \mathrm{msec}$. per letter and per interval between letters. The present report extends the previous findings by examining additional irregular display orders with one 10-letter sequence, examines one 20-letter sequence, and also employs a Polarold filtering technique to provide a preliminary assessment as to whether the "sequential blanking" effect which occurs with certain irregular display orders is retinal or central in origin.

\section{Method}

As in the previously mentioned study (Mayzner, Tresselt, \& Cohen, 1966) a computer-based CRT display system was employed to present the input letter strings. This system consists of a PDP-7 digital computer coupled to two CRT display consoles. Input sequences were programmed for display on a Master (Type 340) CRT and also appeared on a Fairchild slave display which is coated with a P24 phospher with a decay time on the order of a few microseconds. All observations were made on the Fairchild display and 10 observers watched all of the input sequences examined in the study. Again distance of observers from the display, fixation points, and room illumination showed no effects on the results, as in the previous study.

Since content of the input sequences was shown not to have any effect on the results obtained in the previous study only one 10-letter sequence and one 20-letter sequence were examined in the present study. The 10-letter sequence was the word "somersault" (the various display orders that were examined are given in Table 1) and the 20-letter sequence was the word "antidisestablishment," which was examined for only one display order, namely, 10, 18, $8,15,6,17,7,3,2,1,20,11,19,5,16,9,14,4,12$, and 13. The numbers just given for "antidisestablishment" and presented in Table 1 show the various display orders that were examined. For example, in Table 1 the display order 24135869710 given under "somersault" means the " $\mathrm{m}$ " was displayed first but in the third position, the first "s" was displayed second but in the first position, the "e" was displayed third but in the fourth position, the "o" was displayed fourth but in the second position, the " $r$ " was displayed fifth but in the fifth position, the "a" was displayed sixth but in the seventh position, etc.

As in the previous study, letter exposure time and inter-letter times were always approximately equal and for the shortest times examined were approximately .25 msec. Observations of all sequences always began at this shortest exposure time and proceeded upward in steps of 2 msec. until approximately a value of $50 \mathrm{msec}$. per letter and per interval between letters had been reached.

\section{Results and Discussion}

Since "sequential blanking" was found with most of the irregular display orders examined previously (Mayzner, Tresselt, \& Cohen, 1966), and was again Table 1. Input Sequence and Display Orders Employed in the Study

\begin{tabular}{cll} 
& & \multicolumn{1}{l}{ Sequence } \\
SOMERSAULT \\
Display & 1. & 24135869710 \\
Orders & 2. & 13579246810 \\
& 3. & 24681013579 \\
& 4. & 10864297531 \\
& 5. & 97531108642 \\
& 6. & 10837924615
\end{tabular}


found for certain irregular display orders in the present study; a Polaroid filtering technique was employed on these irregular display order sequences. Since the results of this technique were uniform for all display orders examined, only a single example will be explained in detail. Specifically, with the word "chair" and the display order 42513, previously examined (Mayzner, Tresselt, \& Cohen, 1966), in the range from 10 to $20 \mathrm{msec}$. per letter and per interval between letters, "chair" appeared as "c-a-r" (dashes indicating the position of the letters that were not perceived). By placing five separate Polaroid filters over the individual letters of the word "chair" on the face of the CRT display and placing two further filters before each eye of $S$ and then properly aligning the axes of polarization, it was possible to present to the right eye the letters " $c$, " " $a$, " and " $r$ " and to the left eye the letters " $h$ " and " $i$ " (the reverse also was examined to balance any eye dominance factors, but the results were always identical). With this presentation mode, if the sequential blanking is occurring retinally, then presenting the blanked and unblanked letters to different eyes should diminish or totally eliminate the effect. On the contrary, the " $h$ " and "i" continued to be totally blanked, strongly suggesting that the "sequential blanking" phenomena have a central rather than a retinal locus.

In Table 1 , two of the six display orders examined in this study (i.e., display orders 1 and 6) continue to show sequential blanking effects in the range from 10 to $20 \mathrm{msec}$. per letter and per interval between letters, and the letter positions which are not perceived have been underlined. Both these display orders show variations in the elements blanked in contrast to our previous study (Mayzner, Tresselt, \& Cohen, 1966). For example, with the order 24135869710 , it is not the first five elements presented that are blanked, but rather the first, third, fifth, sixth, and seventh. Also, we find for the first time three letters in a row that are not perceived. With the one 20-letter sequence that was examined in this study, the size of the blanked gap is even greater. Thus, with the word "antidisestablishment" and the display order $\underline{10}, 18,8,15, \underline{6}, 17, \underline{7}, \underline{3}, \underline{2}, \underline{1}, 20,11,19, \underline{5}, 16, \underline{9}$, $14,4,12$, and 13 (the underlined elements were not perceived), the entire sub-sequence "sest" (i.e., letters presented seventh, third, second, and first, respectively) is blanked. This finding raises the question of just how many adjacent elements can be blanked in a sequence. Also, the finding of 11 elements out of 20 blanked extends the findings of the previous study and continues to suggest that approximately $50 \%$ of the input string will be blanked for certain irregular display orders, whether the sequence is five, 10 or 20 elements in length.

Turning now to display orders 2, 3, 4, and 5 shown in Table 1 , another entirely unexpected effect appears.
In the previous study (Mayzner, Tresselt, \& Cohen, 1966) display orders 24681097531 and 10864213579 disclosed no sequential blanking but rather, in the range from 10 to $20 \mathrm{msec}$. per letter and per interval between letters, simultaneous flows of movement from the two ends to the center with the display order 24681097531 and simultaneous flows of movement outward from the center to the two ends with the display order 10864213579. With display orders 2 and 3 again simultaneous flows of movement are observed, but now from the beginning to the middle and from the middle to the end, while with display orders 4 and 5 simultaneous movement is from the end to the middle and from the middle to the beginning. The new and unexpected effect involves for the time range indicated, for display orders 2 and 3, a very marked and significant spatial displacement of the second "s" in "somersault" $1 / 2$ to 1 in. in magnitude (with letters that are approximately 1 in. in height and width) away from the " $r$ " to the right. With display orders 4 and 5 the " $r$ " is spatially displaced $1 / 2$ to 1 in. away from the "s" to the left. No other letters in the sequences show any displacement effects. These "sequential displacement" effects are, in fact, so pronounced that their exact magnitudes can be measured precisely by placing a ruler against the face of the Fairchild display. An immediate reaction of all observers is to the effect that the display must not be spacing the letters equally; however, this explanation is quickly disproven, for if one covers all letters with masking tape except the " $r$ " and the " $s$," they are immediately perceived as occurring spatially right next to each other. Further, if the letters in these sequences are reduced in size to about $1 / 8$ in. in height and width, the sequential displacement just described completely disappears and a large sequential blanking effect occurs, with "sau" not perveived for display orders 2 and 3 and "mer" not perceived for display orders 4 and 5. Apparently, then, size or intensity (since as size of letters decreases intensity per unit area increases) or both acting jointly may give rise to either "sequential blanking" or "displacement" effects, depending on display order and display input rate. Finally, with display order 6 , shown in Table 1 , with letters about 1 in. in height and width, and input rate fixed at about 10 msec. per letter and per interval between letters, two letters are blanked, " $\mathrm{m}$ " and "l," shown by underlining, as well as a spatial displacement of the second "s" away from the " $r$ " to the right. Thus, in a single display sequence it is possible to demonstrate both effects discovered to date, namely, "sequential blanking" and "sequential displacement."

\section{Reference}

Maymer, M. S., Tresselt, M. E., \& Cohen, A. Preliminary findings on some effects of very fast sequential input rates on perception. Psychon. Sci., 1966, 6, 513-514. 